\title{
Giving women WOICE postpartum: Prevalence of maternal morbidity using the WHO-WOICE instrument
}

\section{Martha Narvaez Lamus}

Fundacion Valle del Lili

\section{Stephanie Lozano}

Fundacion Valle del Lili

\section{Charles MPoca}

Universidade Estadual de Campinas

Jose Paulo Guida

Universidade Estadual de Campinas

Mary Angela Parpinelli

Universidade Estadual de Campinas

Jose Guilherme Cecatti

Universidade Estadual de Campinas

\section{Maria Escobar Vidarte}

Fundacion Valle del Lili

\section{Lale Say}

Organisation mondiale de la Sante

\section{Doris Chou}

Organisation mondiale de la Sante

Maria Laura Costa ( $\square$ mlaura@unicamp.br)

Universidade Estadual de Campinas https://orcid.org/0000-0001-8280-3234

\section{Research article}

Keywords: Maternal Morbidity, mental health, functionality, depression, puerperium, WHODAS 2.0, PHQ-9, GAD-7

Posted Date: May 12th, 2020

DOI: https://doi.org/10.21203/rs.3.rs-26017/v1

License: (c) (1) This work is licensed under a Creative Commons Attribution 4.0 International License.

Read Full License 
Version of Record: A version of this preprint was published at BMC Pregnancy and Childbirth on May 5th, 2021. See the published version at https://doi.org/10.1186/s12884-021-03727-3. 


\section{Abstract}

\section{Background}

There are no accurate estimates of the prevalence of non-severe maternal morbidities. Given the lack of instruments to fully assess these morbidities, the World Health Organization (WHO) developed an instrument called WOICE.

\section{Objective}

To evaluate the prevalence of non-severe maternal morbidities in puerperal women and analyses factors associated to impaired clinical, social and mental health conditions.

Method

A cross-sectional study with 519 postpartum women in a single encounter 6 to 12 weeks postpartum. The WOICE questionnaire included three sections: the first with maternal and obstetric history, sociodemographic data, risk and environment factors, violence and sexual health; The second considers functionality and disability, general symptoms and mental health; and the third includes data on physical and laboratory tests. Data collection was supported by Tablets with REDCAP software. Initially, a descriptive analysis was performed, with general prevalence of all variables contained in the WOICE, including scales on anxiety and depression (GAD-7 and PHQ-9- altered if $\geq 10$ ), functionality (WHODASaltered when 237.4 ) and data on violence and substance use. Subsequently, an evaluation of cases with alterations was performed, with a logistic regression to investigate factors associated to impaired nonclinical and clinical conditions.

Results

517 women were included, majority (54.3\%) multiparous, ages between 20 and 34 years $(65.4 \%)$ and with partner $(75,6 \%)$. Over a quarter had $(26.2 \%)$ preterm birth, however good perinatal outcomes. Around a third $(30.2 \%)$ reported health problems informed by the physician, although more than $80 \%$ considered having good or very good health. About $10 \%$ reported any substance and $5.9 \%$ suffered violence. Anxiety was identified in $19.8 \%$ of cases, depression in $36.9 \%$ and altered functioning in $4.4 \%$ of women. Logistic regression identified that poor overall health rating was associated to increased anxiety/depression and impaired functioning. Having a partner reduces the perception of women on the presence of clinical morbidities.

\section{Conclusion}

During postpartum care, women presented high frequency of anxiety and depression and relevant frequency of substance use and violence. These aspects of women's health need further evaluation and specific interventions to improve quality of care. 


\section{Background}

Among the new objectives of the Sustainable Development Goals for 2030 is to ensure a healthy life and promote well-being for all, including improving maternal health and reducing maternal mortality (1). It has been suggested that, for each maternal death, 20-30 women suffer from some morbidity; however, these numbers are not based on standardized methods of assessment $(2,3)$.

In the last decade, there has been important progress in the study of severe maternal morbidity (SMM), with standard criteria for the identification of potentially life-threatening conditions (PLTC) and Maternal Near Miss (MNM) (4). Nevertheless, there is growing interest in understanding morbidity in a broader way, including non-severe morbidity. Non-severe morbidity are conditions that may influence and affect women's health and well-being; they include impairment of women's physical, sexual or mental health, and the ability to function in certain domains (cognition, mobility, participation in society), and also the image of their body and their economic and social status (5).

In 2012, the World Health Organization (WHO) Maternal Morbidity Working Group (MMWG) initially developed a new definition of maternal morbidity as "Any health condition attributed to the complication of pregnancy and / or childbirth that may have a negative impact on the well-being and / or functionality of women" $(1,5)$. The relevance of such definition is the innovation in capturing broadly the entire spectrum of morbidity, not excluding the well-known severe maternal morbidity conditions, but also including the non-severe morbidity as well (3).

The MMWG further developed an instrument called WOICE, to measure maternal morbidity, focusing on the health and well-being perception that women have about themselves $(1,6,7)$. The main purpose of this instrument is to identify women suffering of non-severe maternal morbidities, allowing professionals to give adequate care to those conditions, which may not be clearly identified during routine care. This instrument also standardizes the measurement of non-severe maternal morbidities, by using a common framework, allowing different settings and regions to share data and provide strong evidences during pregnancy and postpartum period about the condition.

WOICE comprises tools already developed and validated in the literature, and the construction and results of the pilot study have already been published $(6,8)$. The pilot study using WOICE occurred in three different countries: Jamaica, Kenya and Malawi, between 2015 and 2016 (6) in pregnant women (around 28 weeks) and puerperium (between 6 and 12 weeks), mostly in a low risk setting with a total sample of 1490 female participants (750 pregnant and 740 postpartum) (9). It highlighted the high occurrence of non-severe morbidity in those countries, a condition not correctly identified in other studies that focused only severe maternal morbidity.

WOICE instrument is intended to give voice to neglected conditions in routine care. Lack of knowledge about such conditions lead to inadequate care of these women and contributes to possible short and long- term consequences. Those women who are neglected in the puerperium, return to their homes, with unidentified needs, thus impacting life with their family, newborn and spouse (10). 
WOICE represents a new approach towards measuring non-severe maternal morbidity, allowing health professionals to have a broader understanding of women beyond clinical diseases $(10,11)$.

The objective of the present study is to evaluate the prevalence of non-severe maternal morbidity among puerperal women and analyze factors associated with impaired clinical, social and mental health conditions in a middle - income setting using WOICE.

\section{Methods}

This cross-sectional study used a questionnaire (which includes several instruments) developed by the WHO to assess maternal morbidity in its various aspects. The questionnaire was applied at the postpartum outpatient clinic of the University of Campinas, in a single encounter with women from 6 to 12 weeks postpartum. This outpatient clinic is a referral center for women who had delivery at the maternity hospital and cases scheduled include high-risk women, due to a clinical underlying condition or any complication diagnosed during pregnancy or childbirth. The maternity hospital is a referral center for women with conditions such as hypertension, diabetes, preterm labor and preterm rupture of membranes.

WOICE includes several tools that have already been previously translated and adapted to Portuguese. It includes the 12-item version of the World Health Organization's Disability Assessment Schedule (WHODAS 2.0). This tool evaluates the functionality and ability to perform daily tasks (12-14).

WOICE also includes a tool that evaluates mental health, the General Anxiety Disorder 7-item test (GAD-7), and the 9-item Patient Health Questionnaire (PHQ-9), to assess depression, both already adapted to Portuguese $(15,16)$.

To measure substance use and abuse, WOICE includes Alcohol, Smoking and Substance Involvement Screening Test (ASSIST) (17). For sexual satisfaction and sexual and domestic violence, parts of some scores already validated are within the WOICE, such as the Brief Sexual Symptom Checklist for Women (BSSC-W) and some questions from a questionnaire used in the Multi-country Study on Women's Health and Domestic Violence against Women of the WHO $(18,19)$. Only those last two tools were not previously validated into Portuguese versions, however they contributed with only 5 questions on a total of 126 .

Finally, WOICE gathers data on woman's background, current clinical symptoms and physical examination. The name of the tool precisely refers to the importance of not only consulting a woman during pregnancy and postpartum, but also of "listening" to her voice, complaints and needs.

The proposal was approved by the local Institutional Review Board. All women with age higher than 18 years that agreed to participate signed an Informed Consent form before interview. For adolescents (age bellow 18 years at the time of the interview), written consent and parental consent were both waived, due the consideration that a written consent and a parental consent could put the subject at risk, since violence is one of the conditions evaluated by the study, and it is well known that in cases of domestic violence, the perpetrator is often responsible or very close to the adolescent. However, interviews were 
conducted only after clarification and verbal consent, in a reserved room. The local Institutional Review Board of approved this procedure.

The maternal morbidity measurement questionnaire called WOICE was originally developed in English and further translated into the Brazilian Portuguese. The review was conducted by experienced obstetric investigators and the version was tested (pilot interviews) to measure the time of application and then adapt and modify some words to ascertain accurate understanding. In order to ensure the high quality and reliability of the information collected, the researchers were previously trained to ensure adequate use of the tablets and instruments included in the WOICE questionnaire.

Women were recruited sequentially according to their scheduled postpartum visit, during the data collection period (from November 2017 to December 2018). The postpartum outpatient clinic works every week day, a mean of 6 new cases/day, and they are scheduled according to availability. All women were invited and those who agreed to participate were interviewed. Sample size was estimated in 500 participants for convenience sample, as a pilot study, taking into account that the WOICE instrument had not been previously published by the time when data collection was initiated. The only previous study using such instrument presented 250 women during PPC for each considered country (9).

Data collection was supported by tablets (Samsung Galaxy Tab Tablets S3 - Android), with further transmission, verification and storage of data protected to ensure confidentiality. Each interview was around $30-40$ minutes.

Data processing and collection were supported by REDCAP software and later transferred to the SPSS program. The information gathered was stored in a server located in the informatic department of the institution. A descriptive analysis was performed, including socio-demographic data, clinical and obstetric history, as well as the general prevalence of scores of instruments considered for functional and mental health. Continuous variables were presented on mean (M) and Standard Deviation (SD) and categorical variables in percentage (\%) of frequency. An evaluation of abnormal conditions was performed, considering scores $\geq 10$ for anxiety and depression (20). For WHODAS-12, according to a previous study published, dysfunctionality was considered with the score of $\geq 37.4$ (95th percentile as the cutoff point) (12).

Further, a Poisson multiple regression analysis was performed, providing the respective Prevalence Ratio $(\mathrm{PR})$ and $95 \%$ confidence intervals $(\mathrm{Cl})$, considering three models for evaluating factors associated with impaired conditions. The first model considered as outcome abnormal mental health (score $\geq 10$ for anxiety and depression questionnaire), the second model considered abnormal functioning. The predictors tested were: maternal age, marital status, education, literacy, employed, travel time to facility, parity, gestational age, $\mathrm{BMI}(\geq 30 \mathrm{~kg} / \mathrm{m} 2)$, overall health rating, any clinical condition, preexisting conditions, taking any medication. The third model of logistic regression considered impaired clinical condition (women who answered "yes" to the question: "have you been told you have anything wrong or any medical condition?") as outcome. The tested predictors were the same used in the previous models, 
also in addition to impaired mental health, abnormal functioning, substance use, sexual satisfaction and violence.

In order to understand if the same women were at risk of combined alterations in clinical, social, sexual and mental health, we performed also a descriptive analysis of altered conditions and its combinations two by two.

\section{Results}

In the present study, 519 postpartum women were invited to participate, 2 declined and the 2 women provided only sociodemographic data, therefore 515 gave full consent (Fig. 1). The mean age was 28 years, women mostly had a partner, more than $50 \%$ were multiparous, the illiteracy level was less than $2.4 \%$ and most participants had a secondary level of education and were employed. Over one third of the population took 30-60 minutes to arrive from their house to the health service (Table 1).

Clinical conditions were initially considered through the question: "Since childbirth, have you been informed that there is something wrong / some medical condition?" and $30.2 \%$ of the women had a health condition reported by the attending physician, although more than $80 \%$ reported good or very good health. Considering the gestational results, a quarter (26.2\%) had preterm birth, and $58.3 \%$ delivered by cesarean section; however, predominantly with good perinatal outcomes, $95.7 \%$ reported "good baby health" in the postpartum evaluation, with $88.1 \%$ of exclusive breastfeeding (Table 2 ).

Looking into detail in cases of clinical conditions, based on the question: "any pre-existing condition", the majority (51.7\%) reported having a condition before pregnancy and childbirth (Table 2). A list of conditions, classified them as direct and indirect, of which $13.8 \%$ had gestational diabetes, followed by gestational hypertension (13.4\%), preeclampsia (10.7\%), chronic hypertension (8.2\%) and, operative wound infection (1.7\%), as the most prevalent types of diseases (Table 2).

An important approach, besides evaluating pre-existing conditions, was to evaluate the amount of abnormal conditions diagnosed or identified by WOICE. We found that $(53.1 \%)$ had at least one abnormal condition identified by WOICE, a quarter of women (26\%) had two concomitant conditions identified by WOICE and only $4.0 \%$ had no abnormal condition (Table 2 ).

We identified, through the WOICE questionnaire in this group of women, the use of substances, asking participants whether they used (cigarettes, alcoholic beverages, marijuana, inhalants, sedatives or sleeping pills, hallucinogens, opioids and/or injectable drugs for non-medical use) and $10.0 \%$ of the participants used some type of substance during pregnancy (Table 3). In this group of questions, we also asked "during pregnancy, someone (friend, relative or anyone) expressed concern about the use of any substance" and $66.7 \%$ expressed such concern, followed by $50 \%$ of women that "tried to reduce or stop consumption of any substance ". 
Around $1 / 3$ of women had already resumed their sex life after giving birth and $89.2 \%$ felt they were satisfied with their sex lives, however $55.6 \%(n=10)$ reported pain during intercourse (Table 3). Around $39 \%$ of the women used contraception and $77.2 \%$ of them were prescribed with a method during their first postpartum care medical visit.

Using the WOICE tool, tool, we explored exposure to domestic and sexual violence by asking participants "whether or not they were afraid of the current partner / most recent spouse or any other person" if the spouse / or any other person who pushed, hit and kicked". In our sample, 5.9\% reported to have suffered violence (Table 3).

As part of the Mental Health assessment of our study, we used the validated scales (PHQ-9 and GAD-7). Abnormal conditions were considered if scores $\geq 10(20,21)$ and almost $20 \%$ of the women had anxiety symptoms, followed by $36.9 \%$ with depressive symptoms. For the evaluation of functionality or ability to perform daily tasks, used WHODAS-12 version 2.0 and verified that the mean score was $10.9( \pm 12.9)$, we found $4.4 \%$ of the women had functional alterations (score $\geq 37.4$ ) (12). (Table 4).

The questionnaire was always performed after the scheduled medical consultation and with no interference in the woman's medical follow-up. However, since some of the questions could potentially lead to unpleasant memories and reveal exposure to violence and substance abuse, additional support was offered. Among the included women, $28.3 \%$ used such support, of those $97 \%$ psychological support and $6.6 \%$ social service support (Table 5 ).

In order to investigate factors associated with impaired functioning, mental health and clinical conditions, we performed three multiple regression analyzes. For the first model, that considered WHODAS $\geq 37.4$ as the outcome, the conditions independently associated with abnormal functioning were the presence of impaired clinical health and increased age. Nevertheless, less education and having a partner were protective conditions towards the report of impaired functioning (Table 6). In model 2, considering as outcome abnormal anxiety and depression (scores $\geq 10$ ), illiteracy and poor overall health rating were associated with increased anxiety/depression. However, parity was protective.

In model 3, the clinical conditions reported by the woman (defined when the woman reported having been informed of a clinical diagnosis after delivery) were considered as outcomes and we identified that having a partner reduces the perception of women on the presence of morbidities by $30 \%$ clinics (Table 6).

To understand if the same women suffer from combined conditions, or if there was a pattern in the combination of abnormal findings, we presented a figure that evaluates each parameter and its combinations. Almost a quarter of the participants presented depression and anxiety (38.6\%), followed by clinical conditions associated to depression (14.6\%), anxiety with clinical conditions (13.3\%) (Fig. 2).

\section{Discussion}


This study represents the continuation of an initiative led by the WHO Maternal Morbidity Working Group (MMWG), and represents the implementation the WOICE 2.0 questionnaire to measure non-severe maternal morbidity for the postpartum (PPC) women considering a broad approach of conditions that can impact maternal health, in a high-risk setting (11).

The pilot study conducted in Jamaica, Kenya and Malawi tested the WOICE in pregnant and postpartum women, for the first time, in a mostly low risk and low-income settings, with a total sample of 1490 women (6). In comparison to their findings, our sample included older, more educated women and mostly women with partners. In the pilot study, $(6.1 \%)$ of the women reported having a health problem informed by the attending physician and in our study, this number was much higher, (over $50 \%)$, with more Csection and preterm birth.

Cesarean section rates are increasing worldwide, with Brazil among the most impressive figures (over $50 \%)(22,23)$. Our sample represents a referral center and there is possible selection bias through postpartum scheduled visits, since mostly complicated cases are the ones followed at the institution, therefore not representing the overall cesarean rate in the institution.

Another marker of high-risk assessment is the rate of prematurity. Preterm birth is the main risk factor for infant morbidity and mortality, not only during the neonatal period but also in childhood, it can affect the cognitive dimensions, physical health and behavior, so it is one of the most important challenges for public health. Brazil has rates of preterm birth around $11.5 \%$ (24).

We evaluated the exposure to violence in the WOICE questionnaire, where we could identify that in this group of women surveyed, $5.9 \%$ of the participants were exposed to some type of violence (domesticsexual). Previous reports showed exposure to domestic violence against women as a global phenomenon and these victims are frequently very familiar with their authors, who are people their of daily life. This violence is accepted as "normal" in many societies of the world (25). Estimates by the WHO say that 1 in 3 women worldwide suffer from physical and / or sexual partner and sexual violence by third parties at some point in their life (26) Violence is a sensitive subject, since women are often afraid to talk about it, because of the possible repercussions. Our findings with low frequency of violence, might reflect such fear of the truth.

The high frequency of breastfeeding in our sample must be highlighted, especially considering the highrisk background and frequency of prematurity. Studies show that one of the priorities of these women is the good development of the baby that is supplied in large part by the mother's milk, thus reducing early weaning (27), this might support such levels of breastfeeding, adding the hospital's active work in campaigns, programs to inform women about the benefits of breastfeeding for the baby.

According to a study carried out in 2017 on the indicators of breastfeeding in Brazil in the last three decades, they have led Brazil to be considered a successful country in the implementation of policies and programs to promote breastfeeding with all the necessary tools, knowing that the breastfeeding is not 
only the responsibility of women, it is also shared with society. The prevalence of exclusive breastfeeding for children under 6 months of age in 2013 was $52.1 \%$ (11).

When considering abnormal conditions evaluated by the WOICE instrument, it was striking to observe less than $5 \%$ of women with none alteration. This supports the understanding of multiple aspects that are able to influence women's wellbeing and that during postpartum, women need multidisciplinary support. As a limitation, we do not have prospective assessment of women, in order to pursue the real impact of gestation throughout the reproductive cycle.

Multiple regression presented that having a partner decreased the women's perception of clinical morbidities and that might just reflect more care and support. Having a clinical diagnosis was an independent factor associated to impaired mental health and functioning. This is expected, but rarely reported in a systematic way. Knowing that clinical conditions can be associated to further impairment can guide interventions and improve care (28) In our sample, there was a significant number of women with complications due to hypertension. It is important to highlight that preeclampsia and eclampsia are major causes of morbidity and mortality, especially in low and middle-income settings $(29,30)$.

It is important to note that $96 \%$ of women reported at least one morbidity evaluated by the WOICE instrument, during pregnancy or postpartum period. Performing regular care, we are most likely underreporting the occurrence of morbidities, if we consider the current WHO maternal morbidity framework. WOICE strengths the need to give voice to women during care: if we do not actively ask, we probably will not diagnose non-clinical and non-severe morbidities. However, if we really want to understand in depth the burden of maternal morbidity, we have to apply instruments that may bring to surface some underlying conditions.

Those conditions may be extremely harmful to women, such as intimate partner violence or substance abuse. However, due to social stigmas, those conditions may be source of shame and not reported in routine care; we cannot consider that a woman with those conditions will undergo a positive pregnancy experience, and we will only conduct it properly if we ask.

Another interesting point of our results is that the majority of women reported good or very good health at the time of the interview. Our study design does not allow us to affirm any cause-consequence relation, however we suppose that such result is a consequence of the perception of good healthcare. Some morbid conditions may have occurred and since solved through the puerperium period (due to timely and adequate care, or even due to luck).

Other important concern regarding our results is that our sample represents a population attended in a high risk setting, and results may not be generalizable for the general obstetric population, or even those followed in low-risk settings. However, it highlights the importance of not only considering clinical morbidities, but also other morbidities, even in women with known underlying disease. 
Postpartum care would need to provide much more than contraceptive method orientation, it needs to ensure the opportunity to promote women's health and well-being, and postpartum visits should include a thorough assessment of physical, social, psychological and mental health (10). It is necessary to know all these dimensions and possibilities of alterations in the puerperium, so that it is possible to establish adequate interventions in each scenario.

An important limitation is that WOICE has not been translated and validated into different languages, as Portuguese, and it may difficult comparisons with data obtained using English version. However, the tool is based on several instruments that have been previously validated, and this should be considered when analyzing its results. Another limitation is that questionnaires were answered through an interview administered by a researcher. This methodology may underreport the occurrence of morbidities, notably drugs consumption and intimate partner violence, however such approach was considered to allow the inclusion of women with low-education level.

More research and studies are needed with this instrument to validate it globally, identifying problems and conditions that are not evaluated in a common medical consultation, improving care for women after childbirth.

\section{Conclusions}

The WOICE-WHO instrument allows for an overall evaluation of maternal morbidity. During postpartum care, women presented high frequency of anxiety and depression and relevant frequency of substance use and violence. These aspects of women's health need further evaluation and specific interventions to improve quality of care.

\section{Abbreviations}

1. ANC Antenatal Care

2. ASSIST Alcohol, Smoking and Substance Involvement Screening Test

3. BSSC-W Brief Sexual Symptom Checklist for Women

4. GAD-7 General Anxiety Disorder 7

5. MNM Maternal Near Miss

6. MMWG Working Group Maternal Morbidity

7. PHQ-9 Patient Health Questionnaire 9

8. PLTC Potentially Life- Threatening Conditions

9. PPC Posnatal Care

10. PR Prevalence Ratio

11. REDCAP Research Electronic Data Capture

12. SD Standard Desviation 
13. SMM Severe Maternal Morbidity

14. SPSS Statistical Package for the Social Sciences

15. WHO World Health Organization

16. WHODAS World Health Organization Disability Assessment Schedule 2.0

\section{Declarations}

\section{Ethics approval and consent to participate}

Data collection started only after approval of the research protocol by the local Research Ethics Committee of the Medical Science School of the University of Campinas.

Written consent to participate was obtained for all women with age higher than 18 years; written consent and parental consent was waived for women with age bellow than 18 years, however verbal consent was obtained for this group before interview. The Research Ethics Committee of the Medical Science School of the University of Campinas approved this approach to adolescents, and considered that this group could be at risk, since violence is one of the conditions evaluated by the study and it is well known that in cases of domestic violence, the perpetrator is often responsible or very close to the adolescents. Because of this condition, the Research Ethics Committee waived written consent and parental consent specifically for this group.

The study was approved by the Research Ethics Committee of the Medical Science School of the University of Campinas, under the number of approval 78497817.0.0000.5404 and opinion number 2.386.001.

\section{Consent for publication}

Not applicable

\section{Competing interests}

The corresponding author, Maria Laura Costa is an Associate Editor of this journal.

None of the other authors have any competing interests.

\section{Availability of data and materials}

The datasets used and/or analysed during the current study are available from the corresponding author on reasonable request.

\section{Funding}

No specific funding for this study. 


\section{Authors' contributions}

CML, CJG and PMA had the initial idea for the sutudy. LMN, LS, CMC and GJP

were responsible for data collection. GJP, LMN, CJG and CML were responsible

for planning the analysis and interpretation of data. $\mathrm{LMN}$ and $\mathrm{CML}$ wrote the first

draft of the paper. VMF,LS, CD helped in the interpretation of the analysis. All

authors read and approved the final manuscript.

\section{Acknowledgements}

"Not applicable" in this section.

\section{References}

1. Chou D, Tuncalp O, Firoz T, Barreix M, Filippi V, von Dadelszen P, et al. Constructing maternal morbidity - towards a standard tool to measure and monitor maternal health beyond mortality. BMC Pregnancy Childbirth. 2016;16:45.

2. Alkema L, Chou D, Hogan D, Zhang S, Moller AB, Gemmill A, et al. Global, regional, and national levels and trends in maternal mortality between 1990 and 2015, with scenario-based projections to 2030: a systematic analysis by the UN Maternal Mortality Estimation Inter-Agency Group. Lancet. 2016;387(10017):462-74.

3. Say L, Barreix M, Chou D, Tuncalp O, Cottler S, McCaw-Binns A, et al. Maternal morbidity measurement tool pilot: study protocol. Reprod Health. 2016;13(1):69.

4. Say L, Souza JP, Pattinson RC. Maternal near miss-towards a standard tool for monitoring quality of maternal health care. Best Pract Res Clin Obstet Gynaecol. 2009;23(3):287-96.

5. Firoz T, Chou D, von Dadelszen P, Agrawal P, Vanderkruik R, Tuncalp O, et al. Measuring maternal health: focus on maternal morbidity. Bull World Health Organ. 2013;91(10):794-6.

6. Barreix M, Barbour K, McCaw-Binns A, Chou D, Petzold M, Gichuhi GN, et al. Standardizing the measurement of maternal morbidity: Pilot study results. Int J Gynaecol Obstet. 2018;141(Suppl 1):10-9.

7. Say L, Chou D. Maternal morbidity: Time for reflection, recognition, and action. Int J Gynaecol Obstet. 2018;141(Suppl 1):1-3.

8. Say L, Barreix M, Chou D, Tunçalp Ö, Cottler S, McCaw-Binns A, et al. Maternal morbidity measurement tool pilot: study protocol. Reprod Health. 2016;13(1):69.

9. Silveira C, Parpinelli MA, Pacagnella RC, Andreucci CB, Ferreira EC, Angelini CR, et al. A cohort study of functioning and disability among women after severe maternal morbidity. Int J Gynaecol Obstet. 2016;134(1):87-92. 
10. McKinney J, Keyser L, Clinton S, Pagliano C. ACOG Committee Opinion No. 736: Optimizing Postpartum Care. Obstet Gynecol. 2018;132(3):784-5.

11. Boccolini CS, Boccolini PMM, Monteiro FR, Venancio SI, Giugliani ERJ. Breastfeeding indicators trends in Brazil for three decades. Rev Saude Publica. 2017;51:108.

12. Mayrink J, Souza RT, Silveira C, Guida JP, Costa ML, Parpinelli MA, et al. Reference ranges of the WHO Disability Assessment Schedule (WHODAS 2.0) score and diagnostic validity of its 12 -item version in identifying altered functioning in healthy postpartum women. Int J Gynaecol Obstet. 2018;141(Suppl 1):48-54.

13. Silveira C, Parpinelli MA, Pacagnella RC, Camargo RS, Costa ML, Zanardi DM, et al [Cross-cultural adaptation of the World Health Organization Disability Assessment Schedule (WHODAS 2.0) into Portuguese]. Rev Assoc Med Bras (1992). 2013;59(3):234 - 40.

14. Silveira C, Cecatti JG. Validation of the 36 -item version of the WHO Disability Assessment Schedule 2.0 (WHODAS 2.0) for assessing women's disability and functioning associated with maternal morbidity. Rev Bras Ginecol Obstet. 2017;39(2):44-52.

15. Sousa TV, Viveiros V, Chai MV, Vicente FL, Jesus G, Carnot MJ, et al. Reliability and validity of the Portuguese version of the Generalized Anxiety Disorder (GAD-7) scale. Health Qual Life Outcomes. 2015;13:50.

16. Santos IS, Tavares BF, Munhoz TN, Almeida LS, Silva NT, Tams BD, et al. [Sensitivity and specificity of the Patient Health Questionnaire-9 (PHQ-9) among adults from the general population]. Cad Saude Publica. 2013;29(8):1533-43.

17. Mostardinha AR, Bártolo A, Bonifácio J, Pereira A. [Validation of The Alcohol, Smoking and Substance Involvement Screening Test (ASSIST) Among University Students]. Acta Med Port. 2019;32(4):279-88.

18. WHO I. The ASSIST project - Alcohol, Smoking and Substance Involvement Screening Test. WHO. 2018.

19. WHO | WHO multi-country study on women's health and domestic violence against women. WHO. 2005.

20. Kroenke K, Spitzer RL, Williams JB. The PHQ-9: validity of a brief depression severity measure. J Gen Intern Med. 2001;16(9):606-13.

21. Spitzer RL, Kroenke K, Williams JB, Lowe B. A brief measure for assessing generalized anxiety disorder: the GAD-7. Arch Intern Med. 2006;166(10):1092-7.

22. Ramires de Jesus G, Ramires de Jesus N, Peixoto-Filho FM, Lobato G. Caesarean rates in Brazil: what is involved? Bjog. 2015;122(5):606-9.

23. Nakamura-Pereira M, do Carmo Leal M, Esteves-Pereira AP, Domingues RM, Torres JA, Dias MA, et al. Use of Robson classification to assess cesarean section rate in Brazil: the role of source of payment for childbirth. Reprod Health. 2016;13(Suppl 3):128.

24. Leal MD, Esteves-Pereira AP, Nakamura-Pereira M, Torres JA, Theme-Filha M, Domingues RM, et al. Prevalence and risk factors related to preterm birth in Brazil. Reprod Health. 2016;13(Suppl 3):127. 
25. Garcia-Moreno C, Jansen HA, Ellsberg M, Heise L, Watts $\mathrm{CH}$. Prevalence of intimate partner violence: findings from the WHO multi-country study on women's health and domestic violence. Lancet. 2006;368(9543):1260-9.

26. WHO. I Violence against women. WHO. 2016.

27. Lamounier JA. [Breastfeeding in preterm infants: public health policy in primary care]. Rev Paul Pediatr. 2016;34(2):137-8.

28. Firoz T, McCaw-Binns A, Filippi V, Magee LA, Costa ML, Cecatti JG, et al. A framework for healthcare interventions to address maternal morbidity. Int J Gynaecol Obstet. 2018;141(Suppl 1):61-8.

29. Giordano JC, Parpinelli MA, Cecatti JG, Haddad SM, Costa ML, Surita FG, et al. The burden of eclampsia: results from a multicenter study on surveillance of severe maternal morbidity in Brazil. PLoS One. 2014;9(5):e97401.

30. Zanette E, Parpinelli MA, Surita FG, Costa ML, Haddad SM, Sousa MH, et al. Maternal near miss and death among women with severe hypertensive disorders: a Brazilian multicenter surveillance study. Reprod Health. 2014;11(1):4.

\section{Tables}

Due to technical limitations, Tables 1-6 are only available as a download in the supplemental files section

\section{Figures}




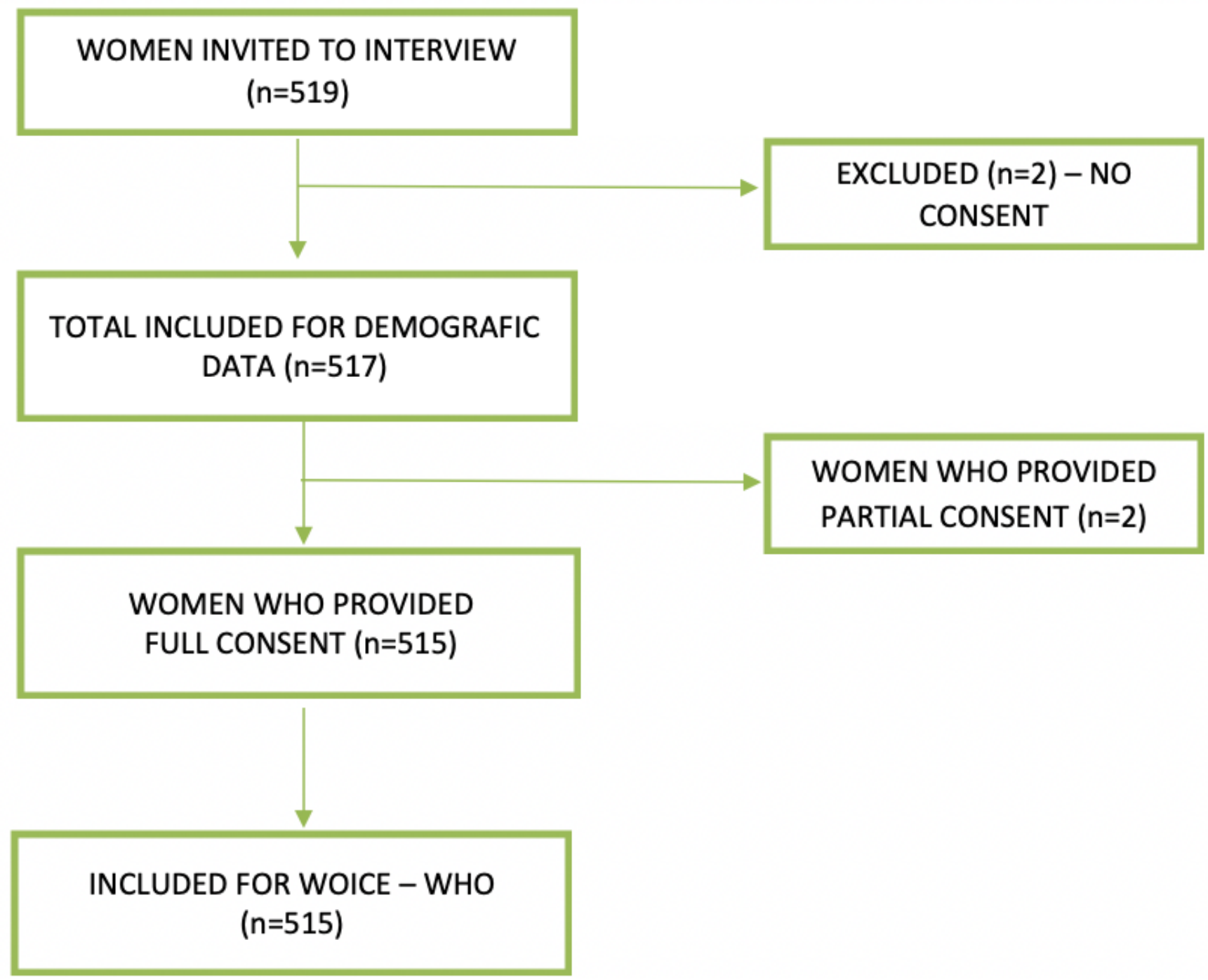

Figure 1

Flowchart of postpartum women included in the study

\section{Supplementary Files}

This is a list of supplementary files associated with this preprint. Click to download.

- MarthaTable3.docx

- MarthaTable5.docx

- MArthaTable6.docx

- STROBEchecklistcrosssectional.doc

- MArthaTable1.docx 
- MarthaTable2.docx

- MarthaTable4.docx 